\begin{tabular}{llll} 
Abstract PTU-042 & Table 1 & & \\
\hline & & Consultant-led & \\
Rockall score & (Mortality \%) & OGD \\
(Mortality \%) & p value \\
\hline 1 & 0 & 0 & n.s. \\
2 & 0 & $*$ & n/a \\
3 & 27.27 & 25 & n.s \\
4 & 35.71 & 12.5 & n.s \\
5 & 25 & 57.14 & n.s \\
6 & 40 & 100 & n.s \\
7 & 42.86 & 0 & n.s. \\
8 & 66.67 & * & n/a \\
\hline *No consultant-led procedures with this Rockall score in the sample. &
\end{tabular}

13 , in patients presenting with haematemesis or melaena and requiring endoscopy. The Rockall score for each patient was calculated and plotted against mortality for the whole population, and then sub classified into consultant- or registrar-performed procedures.

Results 110 procedures performed on 101 patients (41 female, 60 male, median age 71 , age range $23-93$, and an overall mortality of $29.7 \%$ ) were analysed

A direct relationship between an increasing Rockall score and mortality was demonstrated in both the overall population, and also in the registrar-performed procedures, inferring typical population characteristics. Mortality for registrar-performed procedures showed no appreciable difference to that of the overall patient population.

Conclusion Descriptors for achieving competence in therapeutic endoscopy have yet to be defined by the JAG. However, provided local mechanisms for delivering training in, and assessment of, competence at providing endoscopic haemostatic therapy are robust, our findings support the use of a registrar-led out-ofhours GI bleed service.

Disclosure of Interest None Declared.

\section{PTU-043 CAPSULE ENDOSCOPY RETENTION; IS PREDICTION WITHOUT A PATENCY PRE-STUDY POSSIBLE?}

${ }^{1}$ R Makins*, ${ }^{2} \mathrm{R}$ Hopkins. 'Gastroenterology, Cheltenham General Hospital, Cheltenham, UK; ${ }^{2}$ Radiology, Cheltenham General Hospital, Cheltenham, UK

\subsection{6/gutjnl-2014-307263.117}

Introduction Small intestinal capsule endoscopy (CE) is used to investigate occult gastrointestinal bleeding, iron deficiency anaemia, to evaluate the small intestine for Crohn's disease (CD), polyposis syndromes or complications of NSAID use. The only significant complication is capsule retention, either long term or transient, potentially leading to luminal obstruction. The Patency Capsule can be used prior to CE to confirm the likelihood of safe passage however is only for use in conjunction with the system by Given Imaging (Diagmed UK, Personal communication). We use Mirocam CE equipment (Intromedic). Consequently reliance must be placed on radiological studies along with clinical evaluation when assessing the risk of retention. The aim of this study was to determine, in patients where capsule retention had occurred in the setting of a previously reported normal radiological study, whether retrospective review of the radiology could identify stricturing disease and therefore determine whether retention could have been predicted with prior radiological imaging alone.
Methods We reviewed records of all patients who had undergone CE in Gloucestershire since the inception of the local service in 2008. Identified all patients in whom capsule retention occurred. Defined capsule retention as occuring in patients where a stricturing lesion was seen, the capsule appeared not to pass the lesion and colonic mucosa was not positively identified. We retrospectively reviewed any relevant radiological studies performed prior to the CE to determine whether, with hindsight, abnormalities could be identified that would have significantly increased the risk of capsule retention.

Results 465 CE performed locally in Gloucestershire since 2008. CE retention occured above a stricture in 6 (4 male, mean age 45, range 27-61). Indications for CE abdominal pain +/anaemia. In 5 the strictures were thought to be secondary to $\mathrm{CD}$ (past history of CD in 2 only), in 1 due to NSAID use. No stricturing lesions had been identified previously on either barium follow through (2) or cross sectional (CT) imaging (4). 4 studies had been performed locally, 2 by other providers, reported by non-specialist radiologists. On retrospective review significant stricturing lesions were seen on all studies, findings contraindicating CE.

Conclusion $\mathrm{CE}$ is a safe and widely used tool. The risk of capsule retention increases in the setting of Crohn's disease. We have shown that in cases where capsule retention occurred in our institution, where prior radiology was reported as normal, stricturing lesions could be positively identified when reviewed retrospectively by a specialist radiologist. We conclude that thorough radiological assessment is adequate to predict capsule retention and patency studies prior to $\mathrm{CE}$ are therefore unnecessary.

Disclosure of Interest None Declared.

\section{PTU-044 TERTIARY CENTRE EXPERIENCE OF 360 DEGREE SIDE-VIEWING VIDEO CAPSULE ENDOSCOPY}

R Goel*, DM Borrow, KV Patel, I Nasr, M Ward, S Ray, PM Irving, JD Sanderson, SH Anderson. Gastroenterology, Guy's and St Thomas' NHS Foundation Trust, London, UK

\subsection{6/gutjnl-2014-307263.118}

Introduction Since it's development in 1999, video capsule endoscopy (VCE) has become the investigation of choice for examining the small bowel. Recently, a novel panoramic 360 degree side-viewing VCE (Capsovision, Medical Innovations, USA) was launched. It differs from previous capsules in that no data recorder or sensors are required. The images are stored on the capsule itself, which when passed, must be retrieved and sent to the endoscopy reader for analysis.

We report our initial experience of this novel VCE.

Methods We retrospectively analysed the first 51 side-viewing VCE over a 6-month period at our institution. All patients had a clear liquid diet as preparation the day before.

Results 51 patients (26 males) underwent examination with the side-viewing VCE.

39 (76.4\%) examinations were completed and 12 were incomplete. This included 4 which were lost due to being flushed away. Over the same time period, forward-viewing VCE complete results were available in $83.2 \%$ patients.

1 of the incomplete examinations was due to a NSAIDinduced stricture, subsequently diagnosed with a forward-viewing VCE.

31 patients had good bowel preparation, 11 satisfactory preparation and 6 were reported as having poor bowel preparation. 
The duodenal ampulla was reliably identified in 3/47 (6.4\%) examinations. Pathology was identified in $11 / 47$ (23.4\%) examinations.

Conclusion Side-viewing VCE was well tolerated and completed examination results were available for $76.4 \%$ of patients examined. Our findings did not correlate with previous reported results $(71 \%)$ regarding the identification rate of the duodenal papilla as a small bowel landmark with side-viewing VCE.

Advantages of the side-viewing VCE are not needing the patient to wear a recorder, with the data being stored in the capsule itself. This enables multiple patients to be examined on the same day and the number of examinations is not limited by available data recorders. Patients can also take the capsule home and take the capsule at any time which can be useful in the investigation of obscure GI bleeding. Side-viewing VCE is comparable to forward viewing VCE with respect to cost and accuracy.

Disclosure of Interest None Declared.

\section{PTU-045 FLEXIBLE ENDOSCOPIC TREATMENT OF ZENKER'S DIVERTICULUM USING A SOFT DIVERTICULOSCOPE AND AN ENDOSCOPIC KNIFE - VIDEO ABSTRACT}

R Bhattacharyya*, P Bhandari on behalf of Portsmouth research group. Gastroenterology, Portsmouth Hospitals NHS Trust, Cosham, Portsmouth, UK

\subsection{6/gutjnl-2014-307263.119}

Introduction Zenker's diverticulum is a mucosal outpouching through the posterior pharyngeal wall resulting from increased hypopharyngeal pressure. Symptoms may include dysphagia and regurgitation of food. Treatment consists of myotomy of the cricopharyngeus muscle, which is commonly done through an endosurgical approach using a rigid endoscope. We describe a novel method of endoscopic diverticulotomy using a flexible diverticuloscope and an endoscopic knife.

Methods With the patient under general anaesthesia, a guidewire is inserted under direct vision into the oesophageal lumen through a gastroscope. The gastroscope is removed and the guidewire is threaded through a hole on the long flap of the flexible diverticuloscope (ZD overtube, ZDO-22 _ 30; Cook Endoscopy, Winston-Salem, North Carolina). The overtube is gently advanced to $20 \mathrm{~cm}$ until resistance is felt. The gastroscope is then inserted through the overtube and its position adjusted so that the longer flap is in the true oesophageal lumen and the shorter flap is in the diverticulum. The septum is now clearly visualised and stable between the two flaps.

A $3 \mathrm{~mm}$ needle-knife is used to incise the septum. The cut is performed until the muscle fibres are completely cut, and then the cut is extended to a section of the anterior diverticulum and posterior oesophageal wall up to approximately $1 \mathrm{~cm}$ from the bottom. At the end of the procedure, one to three endoclips are placed to prevent perforation or bleeding.

Results We have performed this procedure in 3 patients. 1 patient had no previous surgical intervention, 1 had recurrence following previous surgical diverticulotomy, and 1 patient had severe cervical spondylosis due to which surgery could not be performed as adequate extension of the neck for the surgical procedure was not possible. There have been no complications. Conclusion Endoscopic Zenker's diverticulotomy can be safely performed using a flexible diverticuloscope. We demonstrate the feasibility of performing endoscopic diverticulotomy in patients with recurrence after surgery, or in those who are unsuitable for surgery. We aim to illustrate the principles and technique of this procedure by video demonstration.

Disclosure of Interest None Declared.

\section{PTU-046 MIND OVER MATTER? GASTROINTESTINAL BLEEDING DUE TO THE USE OF NOVEL ORAL ANTICOAGULANTS IN STROKE PREVENTION}

${ }^{1} S$ Beg*, 1J Patel, 'M Bhuva, ${ }^{2} \mathrm{D}$ Collas, ${ }^{1} \mathrm{~B}$ Macfarlane. 'Gastroenetrology, Watford General Hospital, Hertfordshire, UK; ${ }^{2}$ Stroke Medicine, Watford General Hospital, Hertfordshire, UK

\subsection{6/gutjnl-2014-307263.120}

Introduction The occurrence of stroke secondary to the prothrombotic effects of AF is well recognised, with warfarinisation the established therapy for prevention. This is not without complications and so the introduction of the novel oral anticoagulants (NOAC), Dabigatran a factor IIa inhibitor and Rivaroxaban a Xa inhibitor have been received with enthusiasm. Advantages include single dosing, fewer drug interactions and freedom from monitoring, whilst data has demonstrated non-inferiority with respects to stroke prevention. A reported greater propensity to gastrointestinal (GI) bleeding is concerning given the lack of accepted reversal agents, in a cohort of patients who tend to be elderly with comorbidities. We aim to establish the GI complications associated with NOACs in stroke prevention.

Methods We retrospectively analysed 170 consecutive patients from our TIA clinic, who were found to have AF. They were commenced on either Dabigatran or Rivaroxaban, with a total of 86 patient-years of exposure at the time of analysis. We interrogated our computer-based database to ascertain the incidence of anticoagulation related admissions and subsequent management

Results Our cohort of 170 patients had a mean age of 76 years, with a male to female ratio of 8:9. Dabigatran at $220-300 \mathrm{mg} /$ day was prescribed to $45 \%(\mathrm{n}=77)$, whilst $55 \%(\mathrm{n}=93)$ received Rivaroxaban 20-15 mg/day. Rivaroxaban was stopped in one patient due to drug induced hepatitis. One patient was switched from Dabigatran to Rivaroxaban due to dyspepsia.

GI bleeds occurred in $5 \%(\mathrm{n}=4)$ of the Dabigatran group, with 2 upper and 2 lower GI bleeds, occurring on average after 25 days. These patients had a mean age of 84 years, with at least 2 comorbidities. Endoscopy revealed an oesophageal ulcer requiring clipping, new diagnosis of colorectal cancer, haemorrhoids and in one patient no cause was found. GI bleeding occurred in $1 \%(\mathrm{n}=1)$ of the Rivaroxaban group. The patient had a rectal bleed, was 96 years and too frail for investigation, therapy was stopped. The average blood transfusion requirement was 4 units. One individual required resuscitation with 16 units of blood, 12 of fresh frozen plasma, 4 of cryoprecipitate and 1 pool of platelets. Average length of admission was 8.4 days.

Conclusion Existing data suggests that the GI bleed rate whilst on warfarin ranges from $1.3-2.6 \%$, compared to a rate of $2.3-$ $3.6 \%$ in association with NOACs. In our series there was an overall GI bleed rate of $3 \%$, with $1.8 \%$ that could be considered to be significant, requiring blood products and cessation of anticoagulation. Should NOACs become more widely used the lack of established reversal techniques will require local GI units to provide a prompt and skilled bleed service.

Disclosure of Interest None Declared. 